\title{
sciendo
}

\section{Effect of Three Half-Squat Protocols on the Tensiomyographic Twitch Response and Tissue Damage of the Rectus Femoris and the Biceps Femoris}

\author{
by \\ César Berzosa ${ }^{1,2}$, Fernando Sanz-López ${ }^{1,2}$, Oliver Gonzalo-Skok ${ }^{1,2}$, \\ Carlos Valero-Campo ${ }^{1,2}$, José Luis Arjol-Serrano ${ }^{1,2}$, Eduardo Piedrafita ${ }^{1,2}$, \\ Guillermo Aladrén ${ }^{1}$, A. Vanessa Bataller-Cervero ${ }^{1,2}$
}

The aim of this study was to analyse the acute effects of a concentric exercise and two different eccentric overload exercises (EOEs) on blood markers of muscle damage (i.e. creatine kinase [CK], lactate dehydrogenase [LDH], myoglobin [Myo], and malondialdehyde [MDA]) and muscle contractile properties. Ten healthy, young (27 \pm 1.5 years, $179 \pm 6 \mathrm{~cm}, 78.7 \pm 10.8 \mathrm{~kg})$, physically active men $(3.5 \pm 1.9 \mathrm{~h} \cdot \mathrm{w}-1)$ randomly performed three training sessions using the following protocols: a half-squat (HS) as a concentric exercise, and exercises using Versapulley (VP) or YoYo isoinertial technology (YIT) as EOEs (4 $x 7$ repetitions with a 2 min rest interval between sets). Blood samples and tensiomyography measurements were obtained after each training session. Repeated measures analysis of variance (ANOVA) followed by the Tukey test was used to detect differences between the four time points of each variable. The standardized difference or effect size (ES, 90\% confidence limit) in the selected variables was calculated using the basal $S D$. After all exercises, a greater activity of $C K, L D H$, and concentration of Myo, and MDA were found compared to baseline values $(p<0.05)$. A substantially greater activity of $C K, L D H$, and Myo concentration, but not MDA, were found after EOEs when compared to the HS protocol. Substantially lower tensiomyography results in the rectus femoris $(R F)$ were reported, irrespective of the exercise mode performed. Also, no substantial differences were obtained in the biceps femoris (BF) between EOEs and the HS protocol. Time of contraction (Tc) in the RF was possibly to very likely lower in the HS in comparison to EOEs. Additionally, muscular displacement (Dm) in the RF was substantially lower in the HS compared to EOEs. VP produced higher concentrations of damage markers than YIT and concentric exercise did. Furthermore, tensiomyography variables showed similar activation in both exercises, although higher specific fatigue (in the RF) was registered in the traditional HS.

Key words: fatigue, musculoskeletal, performance, strength training.

\section{Introduction}

The best way to improve sports performance may be to understand changes in tissues caused by training. Eccentric overload exercise (i.e. muscles and tendons are elongated during contractions) has been chosen by some researchers due its ability to improve strength and the state of tendons, using squat movement as the preferred exercise for lower limbs (Vázquez-

Guerrero et al., 2016). Despite its efficacy, eccentric overload exercises (EOEs) may alter muscle structure and function to a greater extent than concentric contractions, leading to some discomfort and muscle soreness in athletes performing this type of exercise. Muscle soreness perceived by these athletes could be associated with damage in the muscle fibres after eccentric

1 - Universidad San Jorge. Villanueva de Gállego, Zaragoza. Spain.

2 - ValorA Research Group, Health Sciences Faculty. Universidad San Jorge. 
exercise, normally accompanied by local inflammation and increased lipid peroxidation (LPO), which could cause membrane rupture (Baker et al., 2004; Childs et al., 2001). Damage results in an increase in the concentration of inflammatory markers and different interleukins in the muscle (Buford et al., 2009) as well as the release of intracellular content (e. g. muscle enzymes) to the bloodstream (Conceicao et al., 2012). Consequently, other indirect markers related to muscle damage, such as creatine kinase $(\mathrm{CK})$, lactate dehydrogenase (LDH), and myoglobine (Myo), increase their concentration in the bloodstream (Brancaccio et al., 2010).

Tensiomyography (TMG) is a noninvasive method of assessing changes in the muscle that records muscle reaction to electrical stimulation. Through this method, the degree of fatigue of the muscle can be estimated according to its time of contraction (Tc) or the amplitude of muscular displacement $(\mathrm{Dm})$ in a transverse direction (Rusu et al., 2013). This technique has been used to analyse the neuromuscular system (García-García et al., 2017), fatigue and muscle damage in several sports (Garcia-Manso et al., 2012; Garcia-Garcia et al., 2013; Rodríguez-Ruiz et al., 2012; Gronek et al., 2019). Interestingly, these variables are highly related to Dm values, which are also related to muscle fatigue.

Versapulley (VP) and the YoYo isoinertial technology (YIT) are two devices conceived to develop eccentric overloads. The mechanics of the devices are slightly different, which could affect muscle homeostasis in a different way. VP is based on a rotating cone attached to a flywheel with removable weights (inertial device), and because of that, the force arm is changing for each round, making the resulting overload higher. YIT is based on a rotating cylinder, and the force arm is constant for each round, making the resulting overload more stable.

To the best of our knowledge, no data are currently available on the acute responses to an eccentric overload training (EOT) session with VP and/or YIT devices through the combination of blood markers of muscle damage, plasma concentration variations, and TMG. Therefore, the aims of this study were to 1) analyse changes in the contraction pattern of the muscle, measured by TMG; 2) determine the plasma concentration of malondialdehyde plus 4-hydroxyalkenals (MDA), a frequently used measurement that reflects LPO (Berzosa et al., 2011); and 3) examine the impact of an acute EOT session performed with VP or YIT on blood markers of muscle damage (i.e. CK, $\mathrm{LDH}$, and Myo).

\section{Methods}

The experimental schedule was completed by every participant within 15 days, with at least 72 hours between sessions, as described previously (Coratella et al., 2015). This schedule consisted of 4 testing sessions. Firstly, anthropometry data along with resting blood samples were collected, and basal TMG assessments were conducted between 8:00 and 10:00 am. On the same day (10:00 to 12:00), an incremental test was performed to determine the load that maximized power output (Loadopt) in the barbell half-squat (HS) exercise. The remaining sessions featured random performance of the exercise protocols, i.e. the HS as concentric exercise, the HS using the VP machine, and YIT with the YO-YO squat machine, as eccentric overload exercises. TMG measurements were repeated immediately post-exercise and blood samples were collected 1 hour post-exercise. Participants were instructed not to perform any vigorous exercise 72 hours before each assessment. Furthermore, they were also instructed to avoid drinking coffee or beverages containing caffeine for at least 8 hours before testing.

Every exercise session was carried out at the same time of the day (10:00-12:00) and was preceded by a standardized warm-up, which included $5 \mathrm{~min}$ of jogging and dynamic stretching. After warming up, participants rested 5 minutes before starting testing. The session began with 1 set of 10 repetitions at $50 \%$ of Loadopt and 1 set of 6 repetitions at 75\% Loadopt. Afterwards, participants were asked to perform exercises as quickly as possible, according to their randomized assignment: 1 set of 5 repetitions at $100 \%$ Loadopt in the HS protocol, 1 set of 5 repetitions in the VP and 1 set of 5 repetitions in the YIT protocol.

Venous blood samples were obtained before exercise (basal), and $1 \mathrm{~h}$ after each (HS, VP, and YIT) training session. Blood markers of muscle damage start to rise immediately after exercise and continue to reach a peak after 24-48 $\mathrm{h}$ 
(Brancaccio et al., 2008; Lippi et al., 2008). Therefore, commonly blood samples are taken following this peak, however, in this study, it was considered that changes in the blood markers of muscle damage 1 hour post exercise would allow us to compare the effect of the different exercises. The aim of the study was not to establish the blood kinetics of chosen markers, but to compare the effect of different exercises. Accordingly, no more samples were taken to avoid discomfort to participants. All blood samples were drawn from the antecubital venipuncture and collected into serum-separating tubes. At each sampling time, $10 \mathrm{~mL}$ of blood was collected. Blood samples were immediately centrifuged at $1000 \cdot \mathrm{g}$ for 10 minutes in a Heraeus Primo $\mathrm{R}$ refrigerated centrifuge (Thermo Scientific). Plasma was stored in $250 \mu \mathrm{L}$ aliquots at $-30^{\circ} \mathrm{C}$ until Myo and MDA concentration and enzyme activities were determined.

\section{Participants}

Ten healthy, young $(27 \pm 1.5$ years, $179 \pm 6$ $\mathrm{cm}, 78.7 \pm 10.8 \mathrm{~kg})$, physically active $\left(3.5 \pm 1.9 \mathrm{~h} \cdot \mathrm{w}^{-}\right.$ $\left.{ }^{1}\right)$ males volunteered to participate in this study. All of them were also novice weightlifters. All participants were encouraged not to change their diet during the course of the study. The study conformed to the recommendations of the Declaration of Helsinki. It was also approved by the Aragón Ethical Committee for Clinical Research (protocol reference CP08/2014) on the 21/05/2014. Participants were informed about the nature of the study and were also informed that they could withdraw from the experiment at any time. Written informed consent was obtained from participants before the investigation.

\section{Procedures}

Exercise protocols

The incremental load squat test was performed with participants starting from the upright position with the knees and hips fully extended, stance approximately shoulder-width apart, and the barbell resting across the back at the level of the acromion. Each participant descended in a continuous motion until their thighs were parallel to the floor and then immediately reverted their motion and ascended back to the upright position. Unlike the eccentric phase that was executed at moderate velocity $\left(\sim 0.50-0.65 \mathrm{~m} \cdot \mathrm{s}^{-1}\right)$, participants were always required to perform the concentric phase as fast as possible. The initial load was set at $20 \mathrm{~kg}$ and was progressively increased by $10 \mathrm{~kg}$ until the attained mean velocity was $<0.90 \mathrm{~m} \cdot \mathrm{s}^{-1}$ (Sánchez-Medina and González-Badillo, 2011). Thereafter, the load was individually adjusted with smaller increments ( 5 to $2.5 \mathrm{~kg}$ ). Three repetitions were executed at average speeds $>0.90 \mathrm{~m} \cdot \mathrm{s}^{-1}$. The test was terminated when the maximum power was lower than for the previous load. Three minutes of passive recovery were taken between each set. Only the best repetition at each load, according to the criterion of fastest mean velocity, was considered for subsequent analysis. A free weight setting using a standard $20 \mathrm{~kg}$ Olympic barbell $\left(1^{\text {st }}\right.$ load) (York International Olympic Training Bar, Cranston, RI, USA) was used for testing. Data were registered by a linear encoder (Ergotest, Langesud, Norway). This device has been widely used to evaluate dynamic muscle work and has achieved good reliability scores in previous reports (Gonzalo-Skok et al., 2013; Vuorimaa et al., 2006). In pilot studies, the Loadopt has also shown good reliability $(\mathrm{ICC}=0.96$ and CV $(\%)=$ $3.70 \%$ ). The exercise protocol proposed by Norrbrand et al. (2008) was adopted. Three different exercises (i.e. HS, VP, and YIT) were performed. Each exercise session consisted of 4 sets of 7 repetitions interspersed by 2 -min between-set rest intervals. In the HS protocol, the load used to execute each set was the Loadopt. Furthermore, one $2.7 \mathrm{~kg}$ flywheel $\left(0.07 \mathrm{~kg} \cdot \mathrm{m}^{2}\right.$ moment inertia) in the YIT and the full weights in VP were utilized, considering that the maximum power output in these devices measured through a rotatory encoder (SmartCoach Power Encoder, SmartCoach Europe AB, Stockholm, Sweden) was within these ranges. Similarly, the concentric phase was performed as fast as possible, whereas the eccentric phase was executed at slower velocity (i.e. $\sim 2$ s). Since the squatting depth influences the acute and chronic responses (Hartmann et al., 2012), the range of motion was matched in each exercise (i.e. until the thighs were parallel to the floor). Two devices were used for EOEs because they allowed more (VP) or less (YIT) freedom of movement and could produce different levels of activation and/or damage.

Blood markers

CK activity was determined using a NAC Kinetic-UV kit (Spinreact). It is based on the reaction where phosphocreatine (PCr) and 
adenosine-diphosphate (ADP) are converted to creatine and adenosine-triphosphate (ATP) by the enzyme CK. The generated ATP is a substrate of hexokinase to generate glucose-6-phosphate, which finally oxidizes nicotinamine dinucleotide phosphate (NADP) in the presence of glucose-6phosphate dehydrogenase. The rate of transformation of NADP is measured at $340 \mathrm{~nm}$, and it is proportional to the CK activity of the sample. LDH activity was also determined by spectrophotometry using the DGKC Kinetic-UV kit (Spinreact). It is based on the activity of LDH catalysing the reduction of pyruvate by NADP. The rate of diminution of NADP concentration is monitored at $340 \mathrm{~nm}$, and it is proportional to LDH activity of the sample. One CK or LDH enzymatic unit (U) was defined as the amount of the enzyme that catalyses the conversion of 1 $\mu \mathrm{mol}$ of substrate per minute. Myo concentration was determined by a specific Enzyme-Linked ImmunoSorbent Assay (ELISA) (PALEX Medical). The antibody used in this assay was monoclonal and unique for the antigen of the Myo molecule. MDA concentrations $(\mu \mathrm{M})$ were used as an index of the oxidative breakdown of lipids in the plasma (Janero, 1990). In the assay, MDA reacted with $\mathrm{N}$ methyl-2-phenylindole, yielding a stable chromophore with a maximum peak absorbance at $586 \mathrm{~nm}$; 1,1,3,3-tetramethoxypropane was used as a standard.

\section{Tensiomyography}

TMG features were analysed on the biceps femoris (BF) and the rectus femoris (RF) on the right and the left leg. TMG was provided by the supplier (EMF-Furlan, Ljubljana, Slovenia). The TMG measurement protocol was performed under static and relaxed conditions, with the participant in the supine position and the knee fixed at a $120^{\circ}$ angle $\left(180^{\circ}\right.$ corresponding to full extension of the knee) (RF) or in the prone position and the knee fully extended (BF). The measured limb (RF) was positioned on a triangular wedge foam cushion to keep a fixed knee angle. The skin was marked with a dermographic marker at the points where the TMG sensor was placed, according to the protocol of the supplier, and the distances of these points were measured by referring to the bone area (superior pole of the patella) to confirm subsequent measurements. As a standardized protocol, the black electrode was placed distal and the red electrode was placed proximal, in areas of each muscle determined by the protocol and marking this zone for the post-intervention assessment. The sensor at this point was placed with a pressure of $0.7 \mathrm{~N} / \mathrm{mm}^{2}$, perpendicular to the maximum radial displacement of the muscle. Electrical stimulation began at $20 \mathrm{~mA}$, and was increased by $20 \mathrm{~mA}$ to $40 \mathrm{~mA}$ and $60 \mathrm{~mA}$; software collected sensor data of displacement and muscle contraction until the maximum Dm. Each TMG test lasted approximately $12 \mathrm{~min}$. For each participant, two consecutive measurements were applied at the highest amplitude. Rest intervals of $\geq 10$ s were used between consecutive measurements to minimize the effects of fatigue. None of the participants reported discomfort during electrical stimulation. The contractile properties of $\mathrm{Dm}$ and $\mathrm{Tc}$, as the most reliable variables (Martín-Rodríguez et al., 2017), were calculated from the displacement-time curve obtained from software.

\section{Statistical Analyses}

Data are presented as mean \pm standard deviation (SD). Repeated measures analysis of variance (ANOVA) followed by the Tukey's test were used to detect differences between the four moments of each variable. Significance was set at $p<0.05$.

The standardized difference or effect size (ES, $90 \%$ confidence limit) in the selected variables was calculated using the basal SD. Threshold values for Cohen ES statistics were $>0.2$ (small), $>0.6$ (moderate), and $>1.2$ (large) (Hopkins et al., 2009). For between-times comparisons, chances that differences in blood markers and TMG were better/greater (i.e. greater than the smallest worthwhile change, SWC [0.2 multiplied by the between-subject SD, based on Cohen's d principle]), similar or worse/smaller were calculated. Quantitative chances of beneficial/better or detrimental/poorer effect were assessed qualitatively as follows: $<1 \%$, almost certainly not; $1-5 \%$, very unlikely; $5-25 \%$, unlikely; 25-75\%, possible; 75-95\%, likely; $95-$ $99 \%$, very likely; and $>99 \%$, almost certain. A substantial effect was set at $>75 \%$ (Suarez-Arrones et al., 2014). If the chance of having beneficial/better or detrimental/poorer performance was both $>5 \%$, the true difference was assessed as unclear. 
(a)

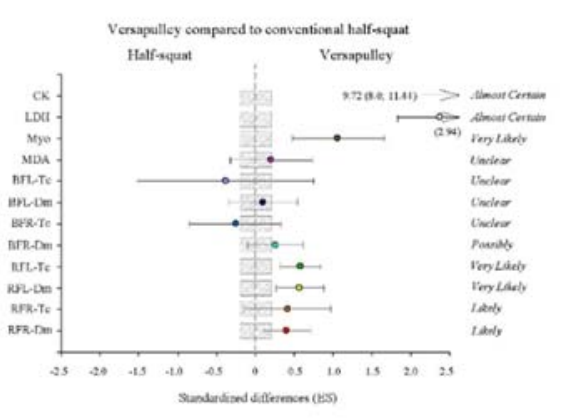

(b)

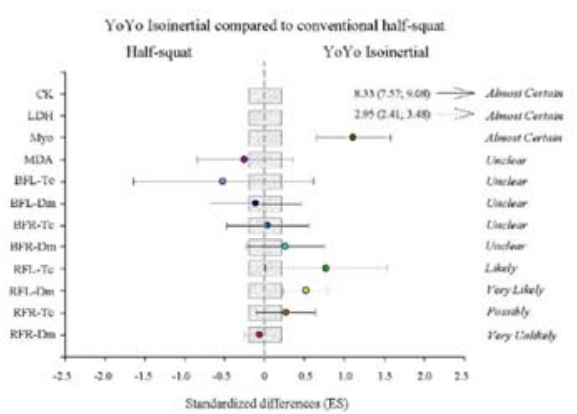

(c)

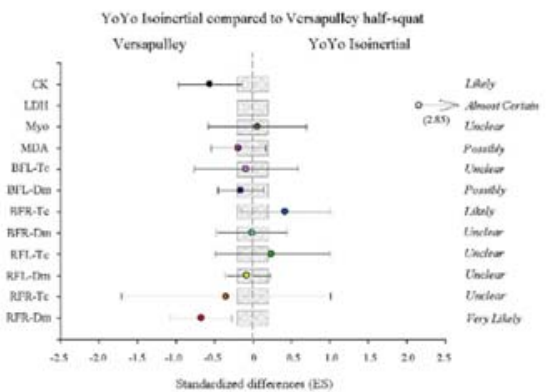

Figure 1. Acute effects of (a) conventional half-squat training compared to Versapulley training, (b) conventional half-squat training compared to YoYo isoinertial training, and (c) Versapulley training compared to YoYo isoinertial training.

Creatine kinase (CK), lactate dehydrogenase (LDH), myoglobine (Myo), malondialdehyde plus 4-hydroxyalkenals (MDA), contraction time in the left biceps femoris (BFL-Tc), muscular displacement in the left biceps femoris (BFL-Dm), contraction time in the right biceps femoris $(B F R-T c)$, muscular displacement in the right biceps femoris (BFR-Dm), contraction time in the left rectus femoris (RFL-Tc), muscular displacement in the left rectus femoris (RFL-Dm), contraction time in the right rectus femoris (RFR-Tc), and muscular displacement in the right rectus femoris (RFR-Dm) (bars indicate uncertainty in the true mean changes with $90 \%$ confidence intervals). Trivial areas were calculated from the smallest worthwhile change (SWC) (see methods). 


\section{Table 1}

Acute changes in muscle damage blood markers, plasma concentration, and tensiomyography following a conventional (HS) half-squat $(n=10)$. Data are mean (SD).

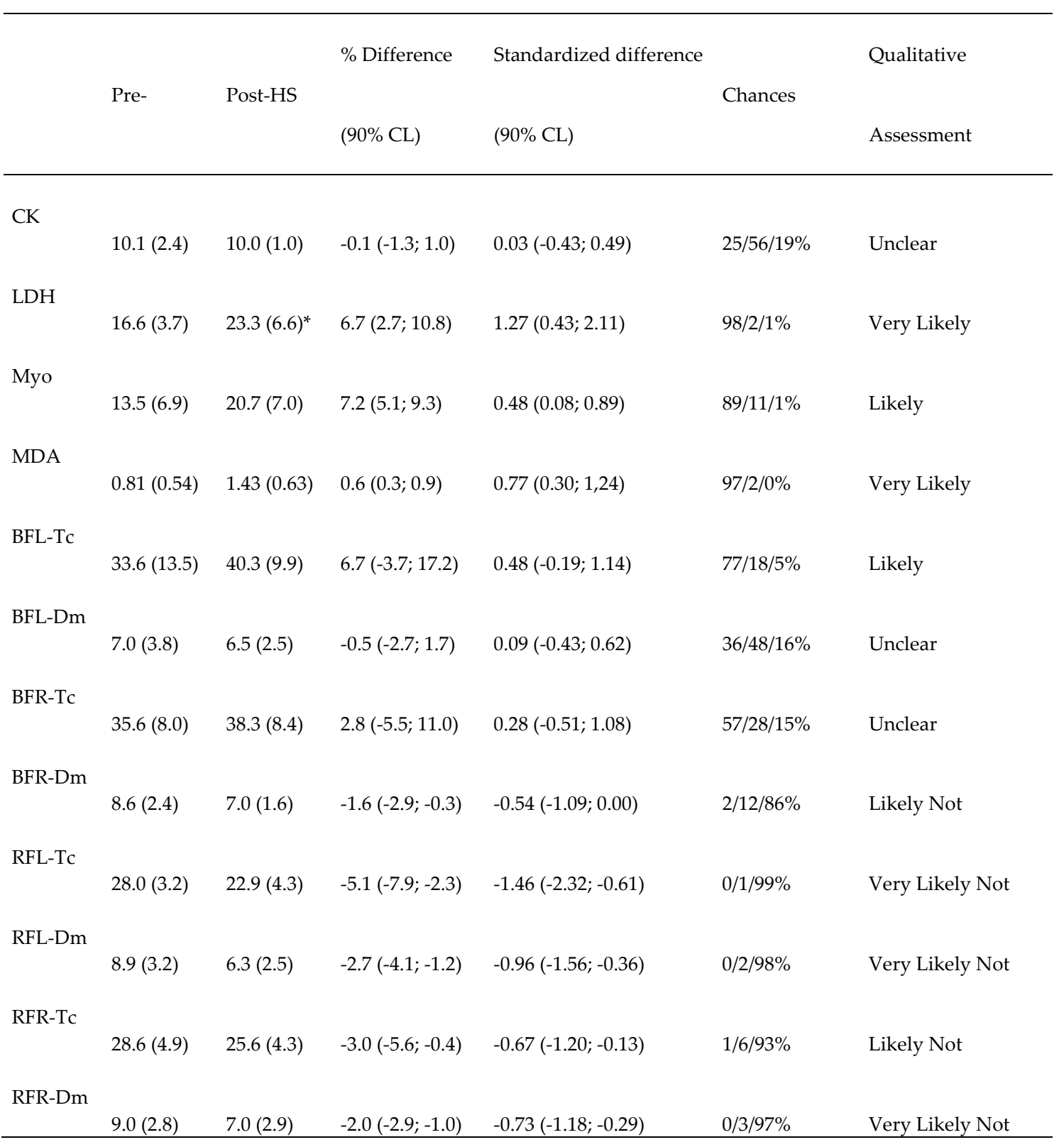

$p<0.05$ versus Pre- $\left.{ }^{*}\right) . C K$ : creatine kinase; LDH: lactate dehydrogenase; Myo: myoglobine; MDA: malondialdehyde plus 4-hydroxyalkenals; BFL-Tc: contraction time in left biceps femoris; BFL-Dm: muscular displacement in the left biceps femoris; BFR-Tc: contraction time in the right biceps femoris; BFR-Dm: muscular displacement in the right biceps femoris; RFL-Tc: contraction time in the left rectus femoris; RFL-Dm: muscular displacement in the left rectus femoris; RFR-Tc: contraction time in the right rectus femoris; RFR-Dm: muscular displacement in the right rectus femoris;\%Difference: percentage difference; Standardized difference: effect size; CL: confidence limits; Chances: percentage chance of having better/similar/poorer values. 
Table 2

Acute changes in muscle damage blood markers, plasma concentration, and tensiomyography following a Versapulley (VP) half-squat $(n=10)$. Data are mean (SD).

\% Difference Standardized difference Qualitative

Pre- $\quad$ Post-VP

Chances

$(90 \% \mathrm{CL})$

$(90 \%$ CL)

Assessment

CK

$10.1(2.4) \quad 32.2(12.2)^{*} \quad 22.6(15.0 ; 30.2) \quad 4.32(3.6 ; 5.04) \quad 100 / 0 / 0 \% \quad$ Almost $C$

LDH

$16.6(3.7) \quad 50.5(4.2)^{*} \quad 33.9(30.1 ; 37.8) \quad 4.44(3.82 ; 5.06) \quad 100 / 0 / 0 \% \quad$ Almost Certain

Myo

$\begin{array}{lllll}13.5(6.9) & 35.0(22.5)^{*} \quad 21.8(7.5 ; 36.0) & 0.77(0.24 ; 1.3) & 96 / 4 / 1 \% & \text { Very Likely }\end{array}$

MDA
$0.81(0.54) \quad 1.85(1.26)^{*}$
$1.3(-0.1 ; 2.8)$
$0.75(-0.09 ; 1.60)$
$90 / 6 / 4 \%$
Likely

BFL-Tc

$33.6(13.5) \quad 38.0(15.3) \quad 4.4(-6.0 ; 14.8) \quad 0.26(-0.33 ; 0.86) \quad 57 / 33 / 9 \% \quad$ Unclear

BFL-Dm

$7.0(3.8) \quad 7.1(2.9) \quad 0.1(-2.1 ; 2.2) \quad 0.14(-0.30 ; 0.58) \quad 40 / 50 / 9 \% \quad$ Unclear

BFR-Tc

$35.6(8.0) \quad 35.9(5.0) \quad 0.4(-6.6 ; 7.3) \quad 0.10(-0.65 ; 0.85) \quad 41 / 36 / 24 \% \quad$ Unclear

BFR-Dm

$8.6(2.4) \quad 7.5(1.6) \quad-1.1(-2.2 ; 0.0) \quad-0.34(-0.79 ; 0.11) \quad 3 / 26 / 71 \% \quad$ Possibly Not

RFL-Tc

$28.0(3.2) \quad 25.8(4.1) \quad-2.2(-4.6 ; 0.2) \quad-0.60(-1.28 ; 0.08) \quad 3 / 12 / 85 \% \quad$ Likely Not

RFL-Dm

$8.9(3.2) \quad 8.1(2.6) \quad-0.8(-1.9 ; 0.2) \quad-0.22(-0.59 ; 0.16) \quad 4 / 43 / 53 \% \quad$ Possibly Not

RFR-Tc

$28.6(4.9) \quad 27.3(2.0) \quad-1.2(-4.2 ; 1.8) \quad-0.21(-0.75 ; 0.34) \quad 10 / 39 / 51 \% \quad$ Unclear

RFR-Dm

$9.0(2.8) \quad 8.2(2.7) \quad-0.7(-1.5 ; 0.0) \quad-0.21(-0.47 ; 0.06) \quad 1 / 47 / 52 \% \quad$ Possibly Not

$p<0.05$ versus Pre- $\left({ }^{*}\right) . C K$ : creatine kinase; LDH: lactate dehydrogenase; Myo: myoglobine; MDA: malondialdehyde plus 4-hydroxyalkenals; BFL-Tc: contraction time in the left biceps femoris; BFL-Dm: muscular displacement in the left biceps femoris; BFR-Tc: contraction time in the right biceps femoris; BFR-Dm: muscular displacement in the right biceps femoris; RFL-Tc: contraction time in the left rectus femoris; RFL-Dm: muscular displacement in the left rectus femoris; RFR-Tc: contraction time in the right rectus femoris; RFR-Dm: muscular displacement in the right rectus femoris;\%Difference: percentage difference; Standardized difference: effect size; CL: confidence limits; Chances: percentage chance of having better/similar/poorer values. 
Table 3

Acute changes in muscle damage blood markers, plasma concentration, and tensiomyography following YoYo isoinertial (YIT) half-squat ( $n=10)$. Data are mean (SD).

\begin{tabular}{llll}
\hline & \% Difference & Standardized difference & Qualitative \\
Pre- $\quad$ Post-YIT & & Chances
\end{tabular}

$(90 \% \mathrm{CL}) \quad(90 \% \mathrm{CL}) \quad$ Assessment

\begin{tabular}{|c|c|c|c|c|c|c|}
\hline & $10.1(2.4)$ & $26.3(4.2)^{*}$ & $16.2(14.3 ; 18.1)$ & $3.54(3.1 ; 3.99)$ & $100 / 0 / 0 \%$ & Almost Certain \\
\hline \multicolumn{7}{|l|}{ LDH } \\
\hline & $16.6(3.7)$ & $61.2(4.7)^{*}$ & $44.6(41.7 ; 47.5)$ & $5.19(4.66 ; 5.72)$ & $100 / 0 / 0 \%$ & Almost Certain \\
\hline \multicolumn{7}{|l|}{ Мyo } \\
\hline & $13.5(6.9)$ & $34.1(8.1)^{*}$ & $20.7(16.8 ; 24.6)$ & $0.82(0.31 ; 1.34)$ & $97 / 2 / 0 \%$ & Very Likely \\
\hline \multicolumn{7}{|l|}{ MDA } \\
\hline & $0.81(0.54)$ & $1.60(1.19)^{*}$ & $0.8(0.0 ; 1.5)$ & $0.65(0.03 ; 1.28)$ & $90 / 9 / 2 \%$ & Likely \\
\hline \multicolumn{7}{|l|}{ BFL-Tc } \\
\hline & $33.6(13.5)$ & $36.0(12.8)$ & $2.4(-5.8 ; 10.5)$ & $0.18(-0.27 ; 0.63)$ & $47 / 45 / 8 \%$ & Unclear \\
\hline \multicolumn{7}{|l|}{ BFL-Dm } \\
\hline & $7.0(3.8)$ & $6.3(2.5)$ & $-0.7(-2.1 ; 0.8)$ & $0.04(-0.34 ; 0.43)$ & $23 / 63 / 14 \%$ & Unclear \\
\hline \multicolumn{7}{|l|}{ BFR-Tc } \\
\hline & $35.6(8.0)$ & $38.4(6.3)$ & $2.8(-4.9 ; 10.5)$ & $0.31(-0.49 ; 1.11)$ & $60 / 27 / 13 \%$ & Unclear \\
\hline \multicolumn{7}{|l|}{ BFR-Dm } \\
\hline & $8.6(2.4)$ & $7.5(1.6)$ & $-1.1(-2.3 ; 0.0)$ & $-0.34(-0.83 ; 0.14)$ & $4 / 26 / 70 \%$ & Possibly Not \\
\hline \multicolumn{7}{|l|}{ RFL-Tc } \\
\hline & $28.0(3.2)$ & $27.1(5.7)$ & $-0.9(-3.6 ; 1.8)$ & $-0.30(-0.97 ; 0.36)$ & $10 / 29 / 61 \%$ & Unclear \\
\hline \multicolumn{7}{|l|}{ RFL-Dm } \\
\hline & $8.9(3.2)$ & $8.0(3.0)$ & $-0.9(-2.1 ; 0.3)$ & $-0.28(-0.67 ; 0.11)$ & $2 / 33 / 65 \%$ & Possibly Not \\
\hline \multicolumn{7}{|l|}{ RFR-Tc } \\
\hline & $28.6(4.9)$ & $26.8(4.1)$ & $-1.8(-4.3 ; 0.8)$ & $-0.37(-0.89 ; 0.14)$ & $3 / 24 / 73 \%$ & Possibly Not \\
\hline \multicolumn{7}{|l|}{ RFR-Dm } \\
\hline & $9.0(2.8)$ & $6.8(2.8)$ & $-2.2(-3.1 ;-1.3)$ & $-0.81(-1.26 ;-0.37)$ & $0 / 1 / 98 \%$ & Very Likely Not \\
\hline
\end{tabular}

$p<0.05$ versus Pre- $\left(^{*}\right)$.CK: creatine kinase; LDH: lactate dehydrogenase; Myo: myoglobine; MDA: malondialdehyde plus 4-hydroxyalkenals; BFL-Tc: contraction time in the left biceps femoris; BFL-Dm: muscular displacement in the left biceps femoris; BFR-Tc: contraction time in the right biceps femoris; BFR-Dm: muscular displacement in the right biceps femoris; RFL-Tc: contraction time in the left rectus femoris; RFL-Dm: muscular displacement in the left rectus femoris; RFR-Tc: contraction time in the right rectus femoris; RFR-Dm: muscular displacement in the right rectus femoris;\%Difference: percentage difference; Standardized difference: effect size; CL: confidence limits; Chances: percentage chance of having better/similar/poorer values. 
Otherwise, we interpreted that change as the observed chance (Hopkins et al., 2009). The PASW Statistics 18 program was used for the statistical analysis and to plot the figures.

\section{Results}

Acute changes in blood markers and TMG from baseline to the HS, VP, and YIT are shown in Tables 1, 2, and 3, respectively. Between-training comparisons are illustrated in Figure 1 . Interestingly, statistically significant differences $(p$ values $<0.05$ ) and substantially greater effect size (very likely to almost certainly) were found between eccentric overload exercises (EOEs) (i.e. VP and YIT) and the HS in plasmatic activities of $\mathrm{CK}, \mathrm{LDH}$, and Myo concentration. MDA did not show substantial differences between EOEs and the HS. Also, results did not show substantial statistical differences in the ANOVA in the BF between EOEs and the HS. Although there were no differences in the ANOVA test in the RF, the Tc was possibly to very likely lower in the HS in comparison to EOEs. In addition, the Dm in the RF was also substantially (likely to very likely) lower in the HS compared to EOEs.

\section{Discussion}

The aim of this study was to analyse the influence of different modes of resistance exercise (i.e., HS, VP, and YIT) on TMG responses and several blood markers of muscle damage and LPO. The main finding was that after acute EOEs (both VP and YIT), muscle mechanical and oxidative damage appeared, which led to an altered muscle contractile capacity. Moreover, VP had a higher impact on muscle damage than YIT. The following experimental evidence supported this view: 1) Tc and Dm in the RF were higher after EOEs in comparison to the HS; 2) the activities of blood CK, LDH, and Myo concentration (markers of muscle damage) were substantially higher after EOEs than after concentric exercise; 3) a substantially higher plasma MDA concentration was found after EOEs and the HS in comparison to baseline; and 4) CK, MDA, Dm, and Tc in the RF were higher when training with VP than with YIT.

Although one of the limitations of this study is the non-homogenous group of study participants, TMG has been included in several sports to analyse different types of muscle contractions (Garcia-Manso et al., 2012; Rey et al., 2012), due to the fact that TMG variables are highly sensitive to muscle fatigue and potentiation (Krizaj et al., 2008). Our results showed that both the HS and EOEs (VP and YIT) yielded lower $\mathrm{Tc}$ and $\mathrm{Dm}$ values in the RF compared to the basal state. Similarly, previous reports have indicated a substantial decrease in Dm values (ES $=5.2)$ after eccentric elbow flexions to achieve exercise-induced muscle damage (EIMD) (Hunter et al., 2012). In this regard, a decrease in Dm after an exercise may indicate an increase in muscle tone and greater muscle stiffness (Hunter et al., 2012). Between-study differences may be due to the exercise type (single-joint vs. multi-joint exercise) or load variables (volume, intensity, or recovery). Regarding Tc, it has been reported that lower values are related to poor tone and a fatigue increase (Krizaj et al., 2008). This could be useful information in the application of warm-up exercise, so as to be careful with the fatigue caused by EOEs. Interestingly, the HS produced a greater Tc decrease in our study, maybe because the metabolic muscle impact of the concentric load is higher than the eccentric load, requiring higher ATP resynthesis and causing muscle fatigue (Nishikawa et al., 2018). The present results differ from previous research in which similar TMG responses (Dm decrease and Tc increase) were found (Hunter et al., 2012). Nevertheless, one previous study found a symmetrical variation (both Dm and Tc increased) in TMG variables (Garcia-Manso et al., 2011). Differences between exercises (cycling vs. squatting) and muscle action (purely concentric vs. eccentric-concentric vs. eccentric overloadconcentric) may be the main explanation for these discrepancies. Collectively, these results suggest that both the HS and EOEs lead to potentiate the RF (Dm decrease), but the HS yields higher fatigue (greater Tc decrease). Nevertheless, further studies are warranted to confirm the present findings.

It has been established that higher activity is produced during EOEs in muscle fibres (Norrbrand et al., 2010, 2011), perhaps due to the activation of different mechanisms not present during concentric action, i.e. actin-titin binding in the presence of calcium, which increases muscle stiffness (Herzog, 2018). Performing this type of 
exercise causes some discomfort (i.e. muscle pain and swelling) (Proske and Morgan, 2001). These symptoms might appear as a result of an injury or when the muscle works over its habitual limits (Proske and Morgan, 2001; Stupka et al., 2001). Furthermore, when muscle fibres are solicited above a critical threshold, muscle tissue can set its break point, resulting in EIMD. At this point, CK, $\mathrm{LDH}, \mathrm{Myo}$, and other intracellular proteins leak into the interstitial fluid and return to the circulation through the lymphatic system (Bijsterbosch et al., 1985). Our results show that EOEs may yield to greater EIMD (i.e. increased $\mathrm{CK}, \mathrm{LDH}$, and Myo) than concentric efforts. These results are in agreement with those found in healthy men after the first training session in a longitudinal study using the YIT (FernandezGonzalo et al., 2014). Interestingly, it has been reported that resistance-trained subjects showed a significantly lower CK response than untrained counterparts (Dolezal et al., 2000). Similarly, CK and $\mathrm{LDH}$ remained at baseline after the last session (Fernandez-Gonzalo et al., 2014). Muscle damage could be higher in untrained than in trained subjects after an isolated eccentric overload session. Therefore, it seems that the participants' training background may be a key factor to determine muscle damage response after EOEs; consequently, training considerations (i.e. sets, repetitions, between-sessions recovery) should be kept in mind when introducing EOEs within strength planning.

In addition to mechanical muscle damage, EOEs may produce oxidative stress. There are several sources of free radicals in contracting muscles, such as oxidase systems associated with membranes, nitric oxide production, and phagocytic processes, which have been proposed to contribute to the overproduction of free radicals during resistance exercises (Powers and Jackson, 2008). Specifically, eccentric contractions are usually accompanied by neutrophil and macrophage infiltration in the tissue. The production of reactive oxygen species and other free radicals by these defensive cells has the potential to damage cell membranes and start a chain reaction called LPO that could finish in muscle cell necrosis (Rodríguez-Ruiz et al., 2012). LPO also alters the structure of lipid bilayer and MDA appears as one of the metabolic products of this reaction, hence muscle MDA may diffuse into the bloodstream, increasing its plasma level, as shown in our study. Nevertheless, substantially higher MDA concentrations were found after performing both EOEs (VP and YIT) and the HS without differences between exercises; the highest concentration was found after VP. These results are in line with those reported after single bouts of exercise (Berzosa et al., 2011).

In conclusion, all these findings could be significant for coaches and athletes who want to incorporate EOEs within their training sessions, because of the increase in muscle damage and the alteration of contractile properties of the muscle. It is also important to note that VP and YIT produce somewhat different responses, and they could be used for different objectives in particular training sessions.

\section{Limitations}

The main limitation of this study is a small sample size. Although we report valuable findings, more participants could provide stronger support and add greater relevance to making an informed decision about the devices used in the training process. Another limitation is that all participants performed a similar volume (hours) of training during the week. Selecting people from the same sport or team could produce findings more relevant to that sport (even though the results might not apply to general training). The last limitation of our study is the age of participants. Caution should be used in applying our conclusions to younger or older athletes, because our design includes only adults of $27 \quad 1.5$ years. Given these limitations, it is obvious that further investigations are needed to ensure the strength of the conclusions.

\section{Practical Implications}

This study has shown that the use of different devices in resistance training may produce different muscle changes depending on the type of the overload (concentric vs. eccentric). Even more, with eccentric overload training, the benefits and/or effects of YIT and VP are slightly different. VP showed higher CK and MDA levels, associated with increased muscle damage, in comparison to YIT and the HS. According to these data, VP and YIT produce a higher impact on muscle, and in our opinion, they should be avoided in beginners and reserved for athletes with extensive background in resistance training. Among these, VP should be reserved for athletes 
who need the most elevated training impact. Having said that, coaches could select the best device to ensure the objectives of their programmed training, knowing that YIT and VP exhibit somewhat different effects.

\section{Acknowledgements}

This work was supported by the Operative Program ERDF Aragon 2014-2020, "Building Europe from Aragon”, Research Group ValorA, Grant No. S08_17R and by Fondo Social Europeo “Construyendo Europa desde Aragón".

\section{References}

Baker JS, Bailey DM, Hullin D, Young I, Davies B. Metabolic Implications of Resistive Force Selection for Oxidative Stress and Markers of Muscle Damage during $30 \mathrm{~s}$ of High-Intensity Exercise. Eur J Appl Physiol, 2004; 92(3): 321-327

Berzosa C, Gómez-Trullén EM, Piedrafita E, Cebrián I, Martínez-Ballarín E, Miana-Mena FJ, Fuentes-Broto L, García JJ. Erythrocyte Membrane Fluidity and Indices of Plasmatic Oxidative Damage after Acute Physical Exercise in Humans. Eur J Appl Physiol, 2011; 111(6): 1127-1133

Bijsterbosch MK, Duursma AM, Smit MJ, Bos OJ, Bouma JM, Gruber M. Several Dehydrogenases and Kinases Compete for Endocytosis from Plasma by Rat Tissues. Biochem J, 1985; 229(2): 409-417

Brancaccio P, Maffulli N, Limongelli FM. Creatine kinase monitoring in sport medicine. Br Med Bull, 2007; 81-82: 209-30

Brancaccio P, Lippi G, Maffulli N. Biochemical Markers of Muscular Damage. Clin Chem Lab Med, 2010; 48(6): 757-767

Buford TW, Cooke MB, Shelmadine BD, Hudson GM, Redd L, Willoughby DS. Effects of Eccentric Treadmill Exercise on Inflammatory Gene Expression in Human Skeletal Muscle. Appl Physiol Nutr Metab, 2009; 34(4): 745-753

Childs, A, Jacobs C, Kaminski T, Halliwell B, Leeuwenburgh C. Supplementation with Vitamin C and NAcetyl-Cysteine Increases Oxidative Stress in Humans after an Acute Muscle Injury Induced by Eccentric Exercise. Free Radic Biol Med, 2001; 31(6): 745-753

Conceicao MS, Libardi CA, Nogueira FR, Bonganha V, Gaspari AF, Chacon-Mikahil MP, Cavaglieri CR, Madruga VA. Effects of Eccentric Exercise on Systemic Concentrations of Pro- and Anti-Inflammatory Cytokines and Prostaglandin (E2): Comparison between Young and Postmenopausal Women. Eur J Appl Physiol, 2012; 112(9): 3215

Coratella G, Milanese C, Schena F. Unilateral Eccentric Resistance Training: A Direct Comparison between Isokinetic and Dynamic Constant External Resistance Modalities. Eur J Sport Sci, 2015; 15(8): 720-726

Dolezal BA, Potteiger JA, Jacobsen DJ, Benedict SH. Muscle Damage and Resting Metabolic Rate after Acute Resistance Exercise with an Eccentric Overload. Med Sci Sports Exerc, 2000; 32(7): 1202-1207

Fernandez-Gonzalo R, Lundberg TR, Alvarez-Alvarez L, de Paz JA. Muscle Damage Responses and Adaptations to Eccentric-Overload Resistance Exercise in Men and Women. Eur J Appl Physiol, 2014; 114(5): 1075-1084

Garcia-Garcia O, Hernandez Mendo A, Serrano Gomez V, Morales-Sanchez V. Application of the Generalizability Theory of Tensiomyography Analysis of Professional Road Cyclists. Revista De Psicologia Del Deporte, 2013; 22: 53-60

García-García O, Serrano-Gómez V, Hernández-Mendo A, Morales-Sánchez V. Baseline Mechanical and Neuromuscular Profile of Knee Extensor and Flexor Muscles in Professional Soccer Players at the Start of the Pre-Season. J Hum Kinet, 2017; 58(1): 23-34 
Garcia-Manso JM, Rodriguez-Ruiz D, Rodriguez-Matoso D, de Saa Y, Sarmiento S, Quiroga M. Assessment of muscle fatigue after an ultra-endurance triathlon using tensiomyography (TMG). J Sports Sci, 2011; 29(6): 619-625

Garcia-Manso JM, Rodriguez-Matoso D, Sarmiento S, de Saa Y, Vaamonde D, Rodriguez-Ruiz D, Da SilvaGrigoletto ME. Effect of High-Load and High-Volume Resistance Exercise on the Tensiomyographic Twitch Response of Biceps Brachii. J Electromyogr Kinesiol, 2012; 22(4): 612-619

Gonzalo-Skok O, Tous-Fajardo J, Arjol-Serrano JL, Mendez-Villanueva A. Determinants, Reliability, and Usefulness of a Bench Press Repeated Power Ability Test in Young Basketball Players. J Strength Cond Res, 2013; 28(1): 126-133

Gronek P, Kryściak J, Cain C.T. Clark, Stroińska W. Exercise for endurance and strength: always separate? TRENDS Sport Sciences, 2019; 3(26): 107-112

Hartmann H, Wirth K, Klusemann M, Dalic J, Matuschek C, Schmidtbleicher D. Influence of Squatting Depth on Jumping Performance. J Strength Cond Res, 2012; 26(12): 3243-3261

Herzog W. Why Are Muscles Strong, and Why Do They Require Little Energy in Eccentric Action? J Sport Health Sci, 2018; 7(3): 255-264

Hopkins WG, Marshall SW, Batterham AM, Hanin J. Progressive Statistics for Studies in Sports Medicine and Exercise Science. Med Sci Sports Exerc, 2009; 41(1): 3-13

Hunter AM, Galloway SD, Smith IJ, Tallent J, Ditroilo M, Fairweather MM, Howatson G. Assessment of Eccentric Exercise-Induced Muscle Damage of the Elbow Flexors by Tensiomyography. J Electromyogr Kinesiol, 2012; 22(3): 334-341

Janero DR. Malondialdehyde and Thiobarbituric Acid-Reactivity as Diagnostic Indices of Lipid Peroxidation and Peroxidative Tissue Injury. Free Radic Biol Med, 1990; 9(6): 515-540

Lippi G, Schena F, Montagnana M, Salvagno GL, Guidi GC. Influence of acute physical exercise on emerging muscular biomarkers. Clin Chem Lab Med, 2008; 46(9): 1313-8

Krizaj D, Simunic B, Zagar T. Short-Term Repeatability of Parameters Extracted from Radial Displacement of Muscle Belly. J Electromyogr Kinesiol, 2008; 18(4): 645-651

Martín-Rodríguez S, Loturco I, Hunter AM, Rodríguez-Ruiz D, Munguia-Izquierdo D. Reliability and Measurement Error of Tensiomyography to Assess Mechanical Muscle Function. J Strength Cond Res, 2017; 31(12): 3524-3536

Nishikawa KC, Lindstedt SL, LaStayo PC. Basic Science and Clinical Use of Eccentric Contractions: History and Uncertainties. J Sport Health Sci, 2018; 7(3): 265-274

Norrbrand L, Fluckey JD, Pozzo M, Tesch PA. Resistance Training Using Eccentric Overload Induces Early Adaptations in Skeletal Muscle Size. Eur J Appl Physiol, 2008; 102(3): 271-281

Norrbrand, L, Pozzo M, Tesch PA. Flywheel Resistance Training Calls for Greater Eccentric Muscle Activation than Weight Training. Eur J Appl Physiol, 2010; 110(5): 997-1005

Norrbrand L, Tous-Fajardo J, Vargas R, Tesch PA. Quadriceps Muscle Use in the Flywheel and Barbell Squat. Aviat Space Environ Med, 2011; 82(1): 13-19

Powers SK, Jackson MJ. Exercise-Induced Oxidative Stress: Cellular Mechanisms and Impact on Muscle Force Production. Physiol Rev, 2008; 88(4): 1243-1276

Proske U, Morgan DL. Muscle Damage from Eccentric Exercise: Mechanism, Mechanical Signs, Adaptation and Clinical Applications. J Physiol, 2001; 537(Pt 2): 333-345

Rey E, Lago-Penas C, Lago-Ballesteros J. Tensiomyography of Selected Lower-Limb Muscles in Professional Soccer Players. J Electromyogr Kinesiol, 2012; 22(6): 866-872

Rodríguez-Ruiz D, Rodríguez-Matoso D, Quiroga ME, Sarmiento S, García-Manso JM, Da Silva-Grigoletto ME. Study of Mechanical Characteristics of the Knee Extensor and Flexor Musculature of Volleyball Players. Eur J Sport Sci, 2012; 12(5): 399-407 
Rusu LD, Cosma GG, Cernaianu SM, Marin MN, Rusu PF, Cioc Nescu DP, Neferu FN. Tensiomyography Method Used for Neuromuscular Assessment of Muscle Training. J Neuroeng Rehabil, 2013; 10(1): 67

Sánchez-Medina L, González-Badillo JJ. Velocity Loss as an Indicator of Neuromuscular Fatigue during Resistance Training. Med Sci Sports Exerc, 2011; 43(9): 1725-1734

Stupka N, Tarnopolsky MA, Yardley NJ, Phillips SM. Cellular Adaptation to Repeated Eccentric ExerciseInduced Muscle Damage. J Appl Physiol, 2001; 91(4): 1669-1678.

Suarez-Arrones L, Tous-Fajardo J, Nunez J, Gonzalo-Skok O, Galvez J, Mendez-Villanueva A. Concurrent Repeated-Sprint and Resistance Training With Superimposed Vibrations in Rugby Players. Int J Sports Physiol Perform, 2014; 9(4): 667-673

Vázquez-Guerrero J, Moras G, Baeza J, Rodríguez-Jiménez S. Force Outputs during Squats Performed Using a Rotational Inertia Device under Stable versus Unstable Conditions with Different Loads. PloS One, 2016; 11(4): e0154346

Vuorimaa T, Virlander R, Kurkilahti P, Vasankari T, Hakkinen K. Acute Changes in Muscle Activation and Leg Extension Performance after Different Running Exercises in Elite Long Distance Runners. Eur J Appl Physiol, 2006; 96(3): 282-291

\section{Corresponding author:}

\section{Dr. César Berzosa}

Universidad San Jorge, Autov. A-23 Zaragoza - Huesca, Km 299, 50.830 Villanueva de Gállego. Zaragoza (Spain).

Phone: (34) 976060100.

Fax: (34) 976077581.

E-mail: cberzosa@usj.es 